\title{
Comparative study on microbiological and histopathological examination of diabetic wounds managed by conventional dressing and vacuum assisted closure therapy
}

\author{
P. Ganesh Kumar ${ }^{1}$, K. Raja ${ }^{2}$, P. Haritha ${ }^{3}$, A. D. Saravanakumari ${ }^{4}$, D. Shanti ${ }^{5}$, S. Vasanthi $^{6}$
}

${ }^{1}$ Department of General Surgery, ${ }^{2}$ Department of Plastic Surgery, ${ }^{3}$ Undergraduate student, ${ }^{4}$ Department of Community Medicine, ${ }^{5}$ Department of Pathology, ${ }^{6}$ Department of Microbiology, Government Villupuram Medical College and Hospital, Villupuram, Tamil Nadu, India

Received: 03 February 2021

Revised: 13 March 2021

Accepted: 16 March 2021

\section{*Correspondence:}

Dr. K. Raja,

E-mail: drskasiraja@gmail.com

Copyright: (c) the author(s), publisher and licensee Medip Academy. This is an open-access article distributed under the terms of the Creative Commons Attribution Non-Commercial License, which permits unrestricted non-commercial use, distribution, and reproduction in any medium, provided the original work is properly cited.

\begin{abstract}
Background: Diabetes mellitus and its complications are rising. Managing the diabetic foot wound is very challenging. Early intervention and intense management using modern technique will improve the diabetic wounds for a definitive procedure and also prevent, reduce the level of amputation of extremity. Our study compares the microbiological and histopathological results of diabetic wounds managed by conventional dressing and VAC (vacuum assisted closure) therapy.

Methods: This was an institutional based prospective study conducted between July 2018 and July 2019 consisting of 50 patients with diabetic wound of lower extremities. Patients were divided into group A- 25 patients underwent conventional dressing and group B- 25 patients underwent negative pressure wound therapy (NPWT). Wound swab and tissue biopsy was obtained on day 0 and day 10 from both groups and compared.

Results: Among patients, $76 \%$ and $24 \%$ were males and females respectively. Histopathologically, necrosis and inflammatory cell infiltrate were significantly decreased in group B compared to group A. Angiogenesis increased notably in group B. On microbiological grounds, from both groups Pseudomonas aeruginosa was found to be the most common organism on day 0 and 10. But the prevalence of infection in group B was grossly reduced when compared to group A.

Conclusions: VAC therapy is a better modality to treat diabetic wound compared to conventional dressing as it accelerates the healing of a chronic wound by increasing angiogenesis and decreasing necrosis, inflammatory cell infiltrate and microbial growth.
\end{abstract}

Keywords: Vacuum assisted closure, Negative pressure wound therapy, Conventional dressing, Diabetic wounds

\section{INTRODUCTION}

Diabetes mellitus and its complications are on the rise in today's world. Managing the diabetic foot wound is very challenging and is one of the major complications of diabetes mellitus. By identifying the pathogenic organism and early salvaging of wound can lead to early intervention with procedures like skin grafting, flap cover and reconstruction, which can also reduce and prevent the level of amputation.

\section{Pathogenesis of diabetic wound}

The body's wound healing mechanism is efficient and works to heal such ulcers. The process involves epithelial regeneration and scar formation. There is intense 
inflammatory reaction with formation of granulation tissue and deposition of extracellular matrix (ECM). ${ }^{1}$ The process involves hemostasis, inflammation, proliferation and remodelling. ${ }^{2}$ The process with a diabetic ulcer is different. It escapes such wound healing mechanisms and is a chronic wound. These factors result in longer time taken to heal and reduce the tensile strength of the wound. ${ }^{3}$ In diabetes mellitus, there are numerous derangements such as ischemia, neuropathy, microangiopathy, impaired immunity and defective angiogenesis. ${ }^{4}$ During hyperglycemia, free radicals also increase which often leads to damage to blood vessels, accelerated atherosclerosis, diabetic nephropathy, neuropathy and retinopathy. ${ }^{5,6}$ Regarding ulcers, neuropathy is a very important complication. Peripheral neuropathy causes loss of sensation predominantly in the lower extremities due to nerve injury and reduced blood flow. This leads to hypoesthesia over the foot which in turn leads to blister formation proceeding to ulcers. They further get infected leading to cellulitis, necrosis and formation of diabetic wound. ${ }^{3}$

Old age, poor glycemic control, diabetic neuropathy, previous history of amputation or ulcers are all risk factors for diabetic ulcers. ${ }^{3}$ Ulcers can be prevented by good glycemic control, detailed examination and proper foot care. ${ }^{7}$ These diabetic ulcers can be treated by conventional dressings or by newer techniques like vacuum assisted closure therapy (VAC) that applies negative pressure to the wound. ${ }^{8}$

Our study is to compare the histopathology and microbiology in diabetic wound management by VAC and conventional dressing method. Early intervention and intense management using modern technique will improve the diabetic wounds for a definitive procedure and also prevent and reduce the level of amputation of extremity.

\section{METHODS}

This was an institutional based randomized controlled trial with convenience sampling conducted for one-year duration from July 2018 to July 2019 consisting of 50 patients with diabetic wound of lower extremities. This was a study for 10 days long with samples collected on day 0 and day 10. Patients were divided into two groups. Group A of 25 patients underwent conventional dressing and group B of 25 patients underwent negative pressure wound therapy (NPWT). Wound swab and tissue biopsy was obtained on day 0 and day 10 from both groups and compared.

Ethical clearance was obtained from the Institutional Ethics Committee before collection of data. Written informed consent was obtained from all the patients before enrolling them for the study. A structured questionnaire was used to enter the demographic and clinical data. 50 consecutive patients with diabetic ulcer (aged between 25 to 65 years; of which 38 were male and 12 were female) were enrolled for study and were randomly separated into two groups.

\section{Inclusion criteria}

Patients aged between 25 years to 65 years (both males and females) who were admitted into the surgery ward with diabetic history, acute and chronic diabetic wound were included in the study.

\section{Exclusion criteria}

Patients with non-diabetic ulcers, malignant ulcers, collagen disorders, neurogenic ulcer, liver cirrhosis, sinus, fistula, patients who were HIV positive or pregnant, florid invasive infection and severe hypoproteinemia were excluded from the study.

On day 0, swab culture was obtained from the wound surface and transported for culture in aerobic medium from both groups and used to obtain microbial colonization results. Wound edge tissue biopsy was obtained from both groups and was fixed in $10 \%$ formalin and dehydrated by a series of increasing concentrations of alcohol. After paraffin block preparations, biopsy sections were stained with hematoxylin and eosin, were studied for evidence of necrosis, presence of inflammatory cell infiltration and presence of angiogenesis in the specimen. ${ }^{9}$

VAC therapy is one that delivers negative pressure to the wound either continuously or intermittently. The VAC (Vacuum assisted closure) dressing includes a polyurethane foam (small- $10 \times 7.5 \mathrm{~cm}$, medium- $12 \times 15$ $\mathrm{cm}$, large $-18 \times 22 \mathrm{~cm}$ ) that is placed according to the size of wound surfaces, which was kept in place with an adhesive drape. A small window was made over the drape which helps in fixing a non-collapsible tube with inbuilt sensor placed on it. Then, the tube was connected to a canister and in turn attached to the VAC machine which provides closed wound dressing. ${ }^{4}$ VAC dressing was applied with Veraflo unit on intermittent mode (with machine on for 5 minutes and off for 2 minutes), medium intensity and negative pressure of $125 \mathrm{mmHg}$ which was usually kept for maximum 2-4 days or until next dressing was changed due to excess discharge or whichever was maximum. VAC therapy applies negative pressure and removes excess of extracellular fluid and decreases tissue edema. This indirectly increases the blood flow to the wound area and improves the wound environment. ${ }^{9}$ An important prerequisite for application of VAC therapy and conventional dressing is the removal of all devitalised tissue from wounds ${ }^{10}$ and a thorough saline wash. In conventional dressing, the wound was cleaned with saline and povidone iodine-soaked gauze was placed over the surface of the wound, this was covered with sterile gauze pads and secured with roller bandages. ${ }^{11}$ On day 10 , again wound swab culture and edge tissue biopsy samples were collected and procedures similar to day 0 techniques were followed to obtain the results. 
Appearance of granulation tissue, reduction in exudate and wound sepsis is faster in VAC therapy than the conventional method. Once the wound bed got adequate granulation the definitive procedures like skin grafting, flaps and reconstruction were performed.

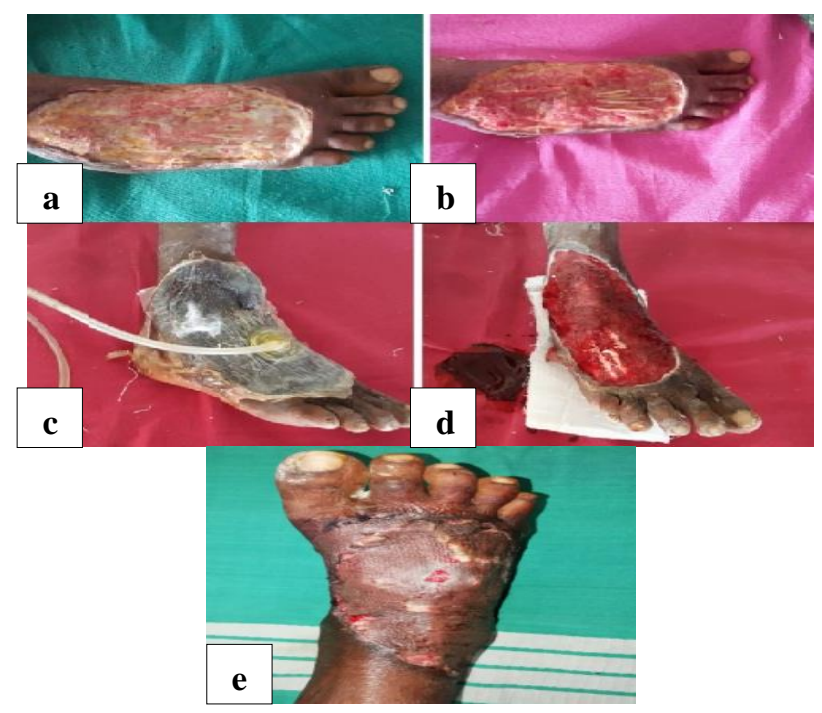

Figure 1: (a) Diabetic wound of a patient on day 0, (b) diabetic wound after debridement, (c) VAC dressing of the diabetic wound, (d) diabetic wound (managed by VAC therapy) on day 10 and (e) wound after graft.

Statistical analysis and data entry were done using statistical package for the social sciences (SPSS) software version 20.0 (IBM, USA). Frequency, mean, standard deviation, standard error was obtained from the data. Independent $t$ test was used to compare means of the two groups (VAC therapy and conventional dressing). $\mathrm{P}<0.05$ was considered significant.

\section{RESULTS}

Of the 50 patients considered for the study, 25 were placed into group A and other 25 into group B. Males were predominant in the study $(76 \% ; 38 / 50)$. All the patients whose data was collected had type 2 diabetes $(100 \%$; $50 / 50)$. The age group of 56 to 65 years had the maximum number of cases $(36 \%, 18 / 50)$. In the ulcers observed, most of them were acute in onset $(36 \% ; 18 / 50)$ followed by traumatic onset $(26 \% ; 13 / 50)$. Most observed ulcers had no discharge $(32 \% ; 16 / 50)$. Maximum number of ulcers had an area of $0-20 \mathrm{~cm}^{2}(40 \% ; 20 / 50)$. The edge of the ulcer was irregular in $58 \%(29 / 50)$ of cases. In most ulcers the surrounding skin was edematous $(74 \% ; 37 / 50)$ and muscle formed its base $(60 \% ; 30 / 50)$. All the cases recorded had signs of inflammation $(100 \% ; 50 / 50)$.

In both the groups, the sex composition was the same. Males were the predominant ones $(76 \% ; 38 / 25)$ compared to females $(24 \% ; 12 / 25)$.

Histopathologically, on day 0, 18 samples showed necrosis in conventional dressing $(36 \%)$ whereas 15 samples from VAC dressing (30\%) showed necrosis. On day 10, necrosis was considerably lesser in wounds with VAC dressing $(10 \% ; 5 / 50)$ compared to those with conventional dressing $(34 \% ; 17 / 50)$ (mean group B versus A, 1.80 versus 1.32 ).

Similarly, in case of inflammatory cell infiltrate, group A with conventional dressing had 19 cases $(38 \%)$ with positive results whereas 20 cases $(40 \%)$ were positive samples in group B with VAC therapy. On day 10, inflammatory cell infiltrate was also lesser in group B with VAC therapy $(10 \% ; 5 / 50)$ compared to conventional dressing $(20 \% ; 10 / 50)$ (mean group B versus A, 1.80 versus 1.60).

Angiogenesis, on day 10, was seen to be higher in group B $(42 \%, 21 / 50)$ compared to group A $(32 \% ; 16 / 50)$ (mean group B versus A, 1.16 versus 1.36).

On microbiological grounds, in group A, Pseudomonas aeruginosa was the most common organism on both day 0 $(10 \% ; 5 / 50)$ and day $10(20 \% ; 10 / 50)$. In group B, on day 0, Pseudomonas aeruginosa, Escherichia coli, Klebsiella species, Staphylococcus aureus $(10 \% ; 5 / 50)$ were all found with the same frequency. While on day 10, Pseudomonas aeruginosa was the most common organism obtained from the swabs $(10 \% ; 5 / 50)$. On day 10 , group A had 8 cases of no growth $(16 \% ; 8 / 50)$ and group B had 19 cases of no growth $(30 \% ; 15 / 50)$ in the swab culture collected.

Table 1: Comparison of various aspects between conventional dressing and VAC therapy.

\begin{tabular}{|c|c|c|c|c|c|c|c|c|}
\hline \multirow{3}{*}{ Parameters } & \multicolumn{4}{|c|}{ Conventional dressing (group A) } & \multicolumn{4}{|c|}{ VAC therapy (group B) } \\
\hline & Day 0 & & Day 10 & & Day 0 & & Day 10 & \\
\hline & $\mathbf{N}(\%)$ & Mean & $\mathbf{N}(\%)$ & Mean & $\mathbf{N}(\%)$ & Mean & $\mathbf{N}(\%)$ & Mean \\
\hline Organisms in swab & $16(32)$ & 4.64 & $17(34)$ & 3.80 & $20(40)$ & 4.00 & $10(20)$ & 5.00 \\
\hline Necrosis & $18(36)$ & 1.28 & $17(34)$ & 1.32 & $15(30)$ & 1.40 & $5(10)$ & 1.80 \\
\hline Inflammatory cells & $19(38)$ & 1.24 & $10(20)$ & 1.60 & $20(40)$ & 1.20 & $5(10)$ & 1.80 \\
\hline Angiogenesis & $10(20)$ & 1.60 & $16(32)$ & 1.36 & $5(10)$ & 1.80 & $21(42)$ & 1.16 \\
\hline
\end{tabular}




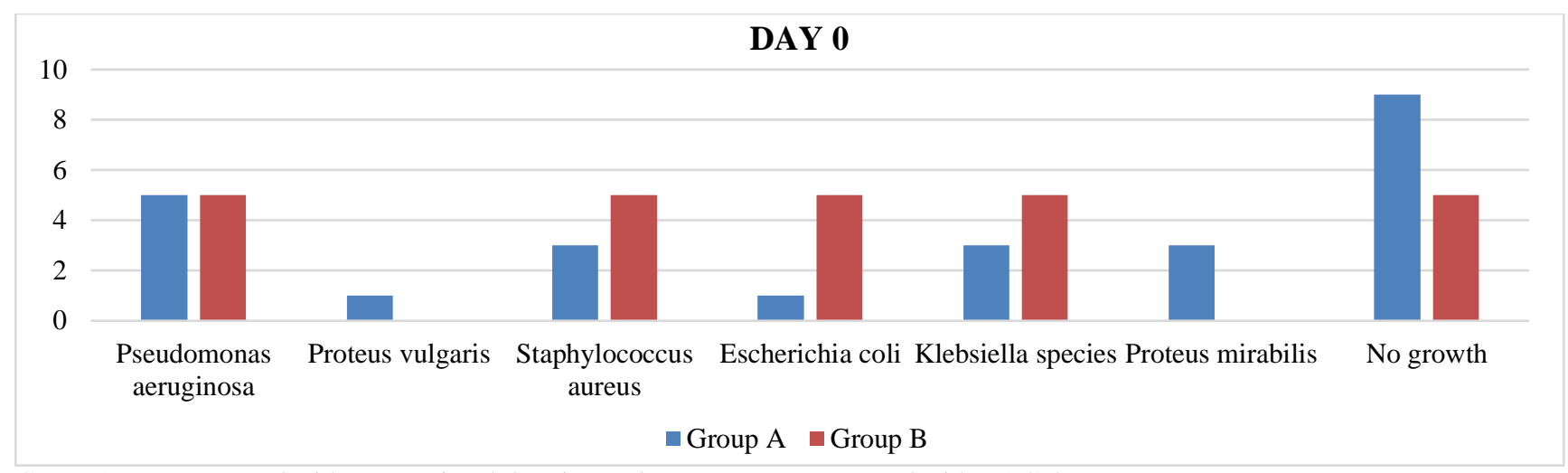

Group A were managed with conventional dressing and group B were managed with VAC therapy

Figure 2: Comparison of microbiological studies on both groups (day 0).

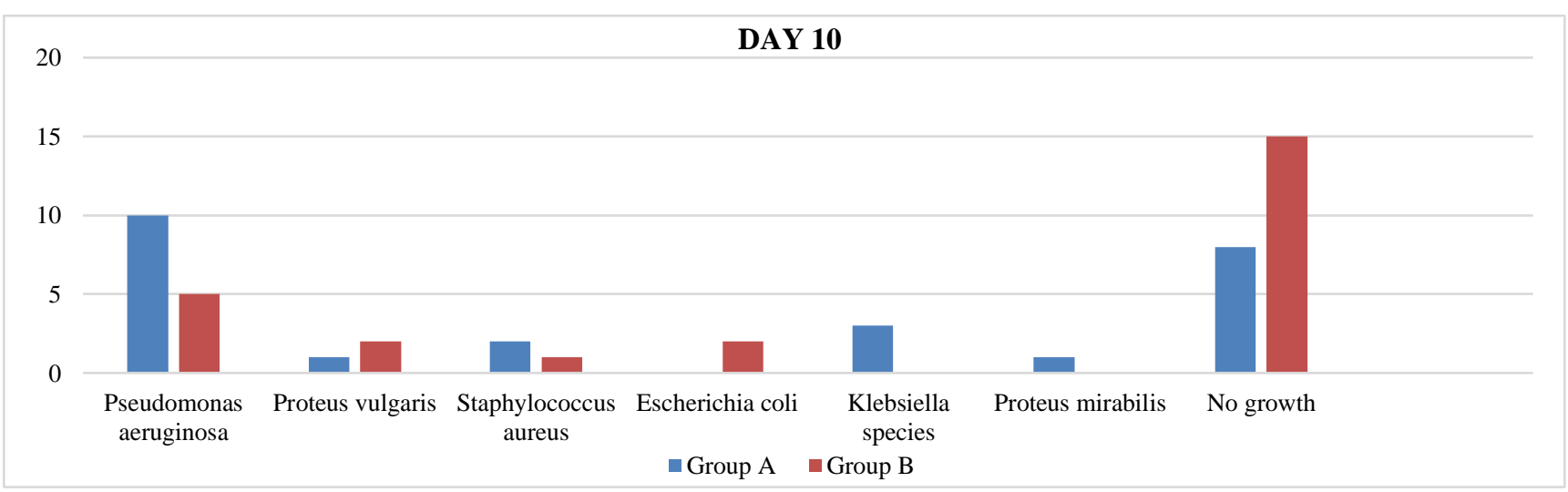

Group A were managed with conventional dressing and group B were managed with VAC therapy

Figure 3: Comparison of microbiological studies on both groups (day 10).

\section{DISCUSSION}

Diabetes mellitus is one of the major chronic diseases. It has also become one of the leading causes of limb loss. About 451 million people (18-99 years) were affected by it in 2017. The number of patients suffering from diabetes is estimated to grow to 693 million by $2045 .^{12}$ India is an epicentre for diabetes and holds the second largest number of patients worldwide. People from the Indian subcontinent make up about $17 \%$ of the total diabetic cases. They are characterised by high insulin resistance and high intra-abdominal fat yet have low body mass index (BMI). This is a predisposing factor for type 2 diabetes mellitus, people suffering from which constitute more than $95 \%$ of total adult diabetic cases. ${ }^{13}$

Such high numbers of diabetic cases point to major risk factors such as genetic predisposition, lifestyle modifications, central obesity and insulin resistance. About $75 \%$ cases of diabetes have genetic predisposition. Urbanisation is also affecting our calorie intake and diet pattern. We Indians also tend to have central obesity predominantly though obesity is less common. We also tend to have more insulin resistance compared to other ethnic groups. ${ }^{14}$
Diabetic foot ulcer is one of the major complications of diabetes mellitus. These ulcers are responsible for more than $30 \%$ hospitalization of diabetic cases. ${ }^{13}$ Among the diabetic patients the prevalence of diabetic foot ulcer is $4 \%$ to $10 \%$ and the lifetime incidence is around $25 \% .{ }^{15}$ Diabetic ulcers are a major risk factor for amputation which affects the patient physically, mentally and socially. Limb amputation occurs 10 to 30 times more often in diabetics compared to non-diabetics and 8 out of 10 non traumatic amputations are due to underlying diabetes. ${ }^{15}$ Thus, finding a better methodology for healing of such ulcers will benefit the patient and the health care system.

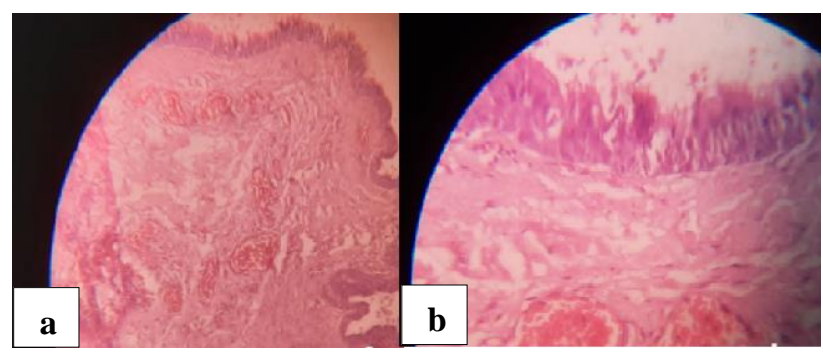

Figure 4: Histopathology of a sample obtained from an ulcer managed by conventional dressing (on day 10) (a) $10 X$ and (b) $100 X$. 
In our study, $76 \%$ patients were males while $24 \%$ patients were female. James et al also had greater number of males than females in both VAC dressing and conventional dressing. ${ }^{11}$ Our study also had increased number of males compared to females. Honnegowda et al reported a greater number of females in VAC therapy group while the group of conventional dressing had more males than females. This study's findings are unlike our results as they have increased number of female patients in the VAC group.
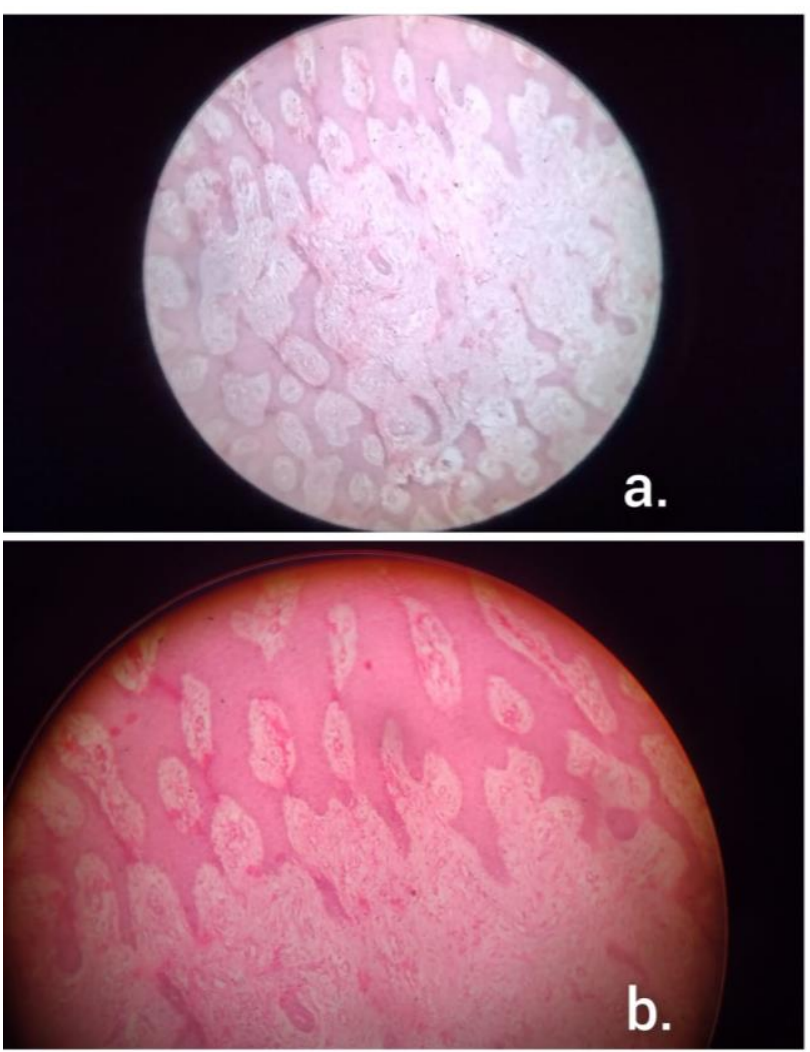

Figure 5: Histopathology of a sample obtained from an ulcer managed by VAC therapy (on day 10) (a) $10 X$ and (b) 40X.

Angiogenesis is the formation of new blood vessels in the wound bed. This results in delivery of more nutrients and oxygen to the growing cells of the wound. ${ }^{9}$ Lone et al also found earlier appearance of granulation tissue in the group managed with VAC therapy. ${ }^{17}$ Armstrong et al found that patients who were managed by VAC therapy grew granulation tissue at a faster rate compared to the group that underwent conventional dressing. ${ }^{18}$ These outcomes are similar to the ones we have obtained from the study. Honnegowda et al also found increased blood flow and blood vessels in patients treated with VAC therapy than in conventional dressing. ${ }^{9}$ Our study also shows similar findings. Zhang et al showed in their study that VAC therapy showed increase in miR-126 in animal models, which regulates VEGF induced angiogenesis. ${ }^{19}$ In our study, angiogenesis also increased considerably in group B $(42 \% ; 21 / 50)$ compared to group A. $(32 \% ; 16 / 50)$ (mean group B versus A, 1.16 versus 1.36 )
Inflammatory cell infiltrate is the range of inflammatory cells that are present at the wound site to mediate the signs of inflammation. Honnegowda et al also found the inflammatory cells to be much lower in NPWT compared to conventional dressing. ${ }^{9}$ Our study also showcased similar results. Huang et al showed that VAC therapy altered the inflammatory cell infiltrate at the wound bed and the inflammation caused by it. $^{20}$ In our study, inflammatory cell infiltration has notably decreased in group B $(10 \% ; 5 / 50)$ than in group A. $(20 \% ; 10 / 50)$ (mean group B versus A, 1.80 versus 1.60 ).

On microbiological ground, Staphylococcus aureus has been reported as the most common organism by James et al. ${ }^{11}$ Nain et al. also found Staphylococcus aureus to be the most common organism in patients treated with NPWT and saw mixed growth in patients with conventional dressing. ${ }^{10}$ Our study did not show similar results as we saw Pseudomonas aeruginosa as the most common organism. Lone et al also found Pseudomonas aeruginosa as the most common organism in patients managed by VAC therapy and conventional dressing. ${ }^{17}$ These findings are similar to our study. Potula et al also found the rate of decrease in patients with growth on day 0 compared to day 16 to be higher in patients with VAC therapy. They commonly isolated Pseudomonas, Proteus, Klebsiella, Escherichia coli, Enterococcus and Staphylococcus aureus from the wounds. ${ }^{3}$ However Armstrong and Lavery reported the presence of infection to be an adverse event. ${ }^{18,11}$ Air leak, concealing an underlying infection, inadequate debridement, bleeding as a culture medium for the organisms are all possible causes of such infection. ${ }^{11}$ Rastogi et al also have compared many such studies on diabetic foot and have found Pseudomonas aeruginosa to be the most common organism to be isolated from such wounds. ${ }^{21}$ In our study, Pseudomonas aeruginosa was the most common organism in conventional dressing and VAC therapy on day 10 . But the prevalence of infection was lower in group B with VAC dressing than group A. 19 patients out of the 25 with VAC therapy had no growth on day 10 compared to only 8 cases of no growth in conventional dressing. Similarly, Nather et al found all their patients treated with VAC therapy, who were positive for microorganisms previously were cleared of infection before surgery was undertaken. ${ }^{4}$ In our study, after VAC therapy none of the patients underwent amputation and in conventional method 2 cases underwent below knee amputation due to uncontrolled wound sepsis with septicaemia, as a lifesaving procedure.

\section{Limitations}

The limitation of the study is that this study did not assess the cost benefit analysis of VAC therapy and conventional dressing. The results of the histopathological and microbiological examination can be improved if the samples are taken on day 5 and day 7 in addition to day 0 and day 10 . 


\section{CONCLUSION}

In conclusion, VAC therapy is a better modality to treat diabetic wound compared to conventional dressing as it accelerates the healing of a chronic wound by increasing angiogenesis and decreasing necrosis, inflammatory cell infiltrate, histopathologically and pathogenic microbial growth. Early intervention and intense management using modern technique (VAC dressing) will definitely improve the diabetic wounds and help in preventing or reducing the level of amputation of extremity.

\section{ACKNOWLEDGEMENTS}

Authors would like to thank Dr. V. Anitha, Vice Principal for examining this project and for sharing her valuable feedback. They would also like to thank ICMR for approving this project under the Short Term Studentship (STS) programme.

Funding: ICMR STS programme

Conflict of interest: None declared

Ethical approval: The study was approved by the Institutional Ethics Committee

\section{REFERENCES}

1. Kumar V, Abbas A, Aster J. Inflammation and repair. In Robbins and Cotran Pathologic Basis of Disease South Asian edition. Elsevier. 2014;108.

2. Guo S, DiPietro LA. Factors Affecting Wound Healing. J Dent Res. 2010;89(3):219-29.

3. Potula VS. Conventional treatment versus vacuum therapy for diabetic foot ulcers treatment. Int Surg J. 2017;5:1.

4. Aziz N, Chionh S, Han A, Chan P, Nambiar A. Effectiveness of Vacuum-assisted Closure (VAC) Therapy in the Healing of Chronic Diabetic Foot Ulcers. Ann Acad Med. 2010;39:353-8.

5. King GL, Loeken MR. Hyperglycemia-induced oxidative stress in diabetic complications. Histochem Cell Biol. 2004;122:333.

6. Kumar V, Abbas A, Aster J. The endocrine system. In Robbins and Cotran Pathologic Basis of Disease South Asian edition. Elsevier. 2014;1105.

7. Wu SC, Driver VR, Wrobel JS, Armstrong DG. Foot ulcers in the diabetic patient, prevention and treatment. Vasc Health Risk Manag. 2007;3(1):65-76.

8. Patmo AS, Krijnen P, Tuinebreijer WE, Breederveld RS. The effect of Vacuum-Assisted Closure on the Bacterial Load and Type of Bacteria: A Systematic Review. Adv Wound Care (New Rochelle). 2014;3(5):383-9.

9. Honnegowda TM, Kumar P, Singh R, Shivakumar S, Rao P, Prasad HK, Kumar S, Kumar U, Udupa EG.
Histopathological study of chronic wounds modulated by intermittent negative pressure therapy under limited access dressing. Plast Aesthet Res. 2015;2:272-6.

10. Nain PS, Uppal SK, Garg R, Bajaj K, Garg S. Role of negative pressure wound therapy in healing of diabetic foot ulcers. J Surg Tech Case Rep. 2011;3:17-22.

11. James SM, Sathasivam S, Thirthar E, Debasis N, Chellappa V, Palanivel C. Comparison of vacuumassisted closure therapy and conventional dressing on wound healing in patients with diabetic foot ulcer: A randomized controlled trial. Nigerian J Surg. 2019;25:14.

12. Kim JL, Shin JY, Roh SG, Chang SC, Lee NH. Factors Affecting Vascular Clogging, Wound Status and Bacterial Culture in Diabetic Foot Ulcers. J Wound Manag Res. 2019;15(2):57-67.

13. Ranjit U, Ranjit A, Viswanathan M. Diabetes mellitus and its complications in India. Nature Rev Endocrinol. 2016;12.

14. Ambady R, Snehalatha C, Vijay V. Burden of type 2 diabetes and its complications-The Indian scenario. Diab Research. 2001;83.

15. Singh N, Armstrong DG, Lipsky BA. Preventing foot ulcers in patients with diabetes. JAMA. 2005;293(2):217-28.

16. Kumar V, Abbas A, Aster J. Cellular responses to stress and toxic insults: Adaptation, injury and death. In Robbins and Cotran Pathologic Basis of Disease South Asian edition. Elsevier. 2014;108.

17. Lone AM, Zaroo MI, Laway BA, Pala NA, Bashir SA, Rasool A. Vacuum-assisted closure versus conventional dressings in the management of diabetic foot ulcers: a prospective case-control study. Diabetic Foot Ankle. 2014;5(1):23345.

18. Armstrong DG, Lavery LA. Diabetic Foot Study Consortium. Negative pressure wound therapy after partial diabetic foot amputation: A multicentre, randomised controlled trial. Lancet. 2005;366:1704-10.

19. Zhang D, Li Z, Wang Z, Zeng F, Xiao W, Yu A. MicroRNA-126: a promising biomarker for angiogenesis of diabetic wounds treated with negative pressure wound therapy. Diabetes Metab Syndr Obes. 2019;12:1685-96.

20. Huang C, Leavitt T, Bayer LR, Orgill DP. Effect of negative pressure wound therapy on wound healing. Curr Probl Surg. 2014;51:301-31.

21. Rastogi A, Bhansali A. Diabetic Foot Infection: An Indian Scenario. J Foot Ankle Surg (Asia-Pacific). 2016;3(2):71-9.

Cite this article as: Kumar PG, Raja K, Haritha P, Saravanakumari AD, Shanti D, Vasanthi S.

Comparative study on microbiological and histopathological examination of diabetic wounds managed by conventional dressing and vacuum assisted closure therapy. Int Surg J 2021;8:1195-200. 